\title{
Structural properties, photoelectric and photoluminescent characteristics of nanostructured silicon
}

\author{
A.I. Luchenko ${ }^{1}$, M.M. Melnichenko ${ }^{2}$, K.V. Svezhentsova ${ }^{1}$ \\ ${ }^{1} V$. Lashkaryov Institute of Semiconductor Physics, NAS of Ukraine \\ 41, prospect Nauky, 03028 Kyiv, Ukraine \\ ${ }^{2}$ Taras Shevchenko Kyiv National University, Physics Faculty \\ 4, prospect Glushkova, 03127 Kyiv, Ukraine; e-mail: realcrystallab@univ.kiev.ua
}

\begin{abstract}
Nanostructured silicon layers (3-60 nm) have been formed upon substrates of monocrystalline silicon with a very large area $\left(100 \mathrm{~cm}^{2}\right)$, multicrystalline and metallurgical silicon by stain etching. We studied optical and structural properties of nanostructured silicon using scanning tunnel microscopy, scanning electron microscopy, Auger electron spectroscopy, secondary ion mass spectrometry, and photoluminescence methods. Results of studying the nanostructured Si properties obtained using the method of chemical processing have confirmed an opportunity to create this multifunctional material with stable characteristics. The authors have developed the sensor systems with use of nanostructured silicon as a sensitive layer, which properties depend on thickness of the obtained layer and are controlled by parameters of the respective technological process. Using the example of the photoluminescent sensor with the nanostructured $\mathrm{Si}$ layer, it has been shown that such a sensor can be successfully used to detect small concentrations of toxins (pesticides phosalone $10^{-8}-10^{-9} \mathrm{~mol} / \mathrm{l}$ ) as well as for specific biological pollutants, such as protein components, polysaccharides, cells worsening the quality of products of biotechnological synthesis.
\end{abstract}

Keywords: stain etching, nanostructured silicon, photodetector, biochemical sensor, biological sensor, photosensitivity, UV sensitivity.

Manuscript received 13.08.12; revised version received 10.09.12; accepted for publication 17.10.12; published online 12.12.12.

\section{Introduction}

In recent years, development of nanotechnologies stimulated interest to study nanocrystalline materials, due to their unique physical and chemical properties. Application of nanocrystalline materials has given a powerful push for development of touch engineering, which at the given stage covers practically all spheres of human life from space engineering to creation of microelectronic biorobots for realization of the most complicated surgical operations.

Vivid examples of these materials are porous, nanoporous and nanostructured types of silicon, which dimension defines behavior of optical and physical properties. On the basis of porous silicon obtained using the method of electrochemical etching, sensors of humidity, gas analyzers, photodetectors, solar elements and light-emitting diodes are created [1-5].

However, as it has been shown in numerous works devoted to application of porous silicon in sensorics, the basic problem is related to instability of their physical and optical characteristics. It is necessary to note that the properties of nanostructured silicon obtained by the chemical etching method applied to monocrystalline silicon from the viewpoint of applying them in touch systems practically are not investigated. 
At formation of nanostructured silicon on substrates of monocrystalline silicon there is a change not only in structural properties, which results in change of the bandgap width and occurrence of quantum size effects, but also in formation of new silicon compounds on a surface with the increased contents of hydrogen and amorphous silicon. This complex structure results in occurrence of new electrophysical, photoelectric, thermophysical, electro- and photoluminescent properties. To create sensors, first of all it is necessary to create a stable layer of nanostructured silicon with controlled properties and ability to optimize them for specific applications in touch systems, and this was a subject of researches in this work.

\section{Experimental}

We used boron doped monocrystalline silicon square wafers with resistivity of $1 \mathrm{Ohm} \cdot \mathrm{cm}$, with area of $100 \mathrm{~cm}^{2}$ and thickness of $0.3 \mu \mathrm{m}$, multi-crystal silicon square wafers with the same area and metallurgical silicon. Nanostructured layers were prepared using stain etching in $\mathrm{HF}: \mathrm{HNO}_{3}$ solution at room temperature, natural day-time illumination and time duration from 1 up to $20 \mathrm{~min}$. The thickness $(d)$ of a layer of nanostructured silicon was changed from 3 up to $60 \mathrm{~nm}$, which was set by parameters of the technological process at chemical modification on a surface of monocrystalline silicon and determined by Auger electron spectroscopy (AES).

Detailed studying the chemical composition of nanostructured Si surface was carried out by the method of AES by using LAS-2000 installation intended for surface investigation. The spectra were recorded with the energy resolution $3.4 \mathrm{eV}$. Energy of the primary electron beam was $3 \mathrm{keV}$, and probe current was $5 \cdot 10^{-7} \mathrm{~A}$. The information was taken from the area of $100 \times 100 \mu \mathrm{m}$. To investigate element distribution over the depth of the samples under investigation, the latter were etched using the argon ion beam with the energy $4 \mathrm{keV}$. The etching rate was $30 \AA / \mathrm{min}$. The specify the chemical composition of samples surface and determine the types of present chemical compounds, the research of samples was carried out using the method of secondary ion-mass spectroscopy (SIMS) making use of a quadrupole massspectrometer. The speed of etching during analysis of chemical composition was $3 \AA / \mathrm{min}$. The structure of nanostructured surface was studied using a scanning tunnel microscope (STM) and scanning electron microscope (SEM). Photoluminescence and reflection spectra were studied in detail earlier [6].

To study photoelectric characteristics of the samples with nanostructured surface, we used the following heterostructure: metal - nanostructured silicon - monocrystalline silicon - metal (Fig. 1). Contacts with the area $1 \mathrm{~mm}^{2}$ and thickness $0.3 \mu \mathrm{m}$ were prepared using the method of magnetron dispersion of $\mathrm{Ti}-\mathrm{Ni}$ with using of a metal mask. The back contact was continuous and had thickness $0.3 \mu \mathrm{m}$.

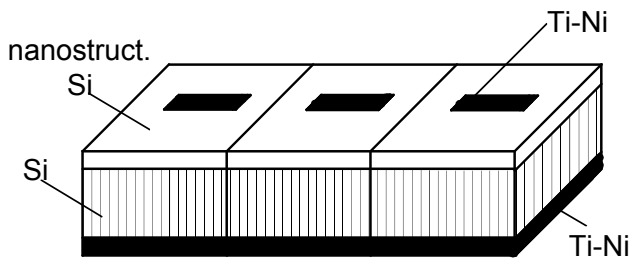

Fig. 1. Heterostructure: metal - nanostructured silicon monocrystalline silicon - metal.

\section{Results and discussion}

\subsection{Structural properties}

Study of the surface morphology by using SEM and STM has shown that nanostructured $\mathrm{Si}$ has an ordered structure and exactly repeats the morphology of the surface of monocrystalline silicon substrates, forming pores on every separate relief piece. The method of STM was used for detailed studying the separate pores of nanostructured surface (Fig. 2). Analysis of the obtained images shows that nanostructured $\mathrm{Si}$ surface is regularly covered with nanoscale hills up to $20 \mathrm{~nm}$ in height. However, owing to certain sizes and tips, the information of the depth can be incorrect as deep narrow pores can be displayed as shallow holes. Therefore, the thickness of nanostructured Si layers has been studied additionally by AES method. The thickness of layers was determined as a product of the speed of layer-bylayer argon ion etching ( $3 \mathrm{~nm} / \mathrm{min})$ and time of etching.

During formation of nanostructured silicon layer, both contents, and in-depth distribution of oxygen $(\mathrm{O})$, carbon $(\mathrm{C})$ and oxidized silicon $\left(\mathrm{SiO}_{\mathrm{x}}\right)$ change. Fig. 3a shows the dependence of concentration of $\mathrm{SiO}_{\mathrm{x}}$ complexes on time of chemical etching obtained by the AES method. It can be seen that with increasing the time of etching, $\mathrm{SiO}_{\mathrm{x}}$ contents both on a surface and in depth of a layer grow, and the maximal quantity of $\mathrm{SiO}_{\mathrm{x}}$ at the surface is observed for samples prepared by etching for 5 min (curve 3). The further increase of time of chemical etching results in insignificant reduction of the content of $\mathrm{SiO}_{\mathrm{x}}$ complexes at the surface (curve 4), which can be related with gradual etching out of the nanostructured $\mathrm{Si}$ layer. Dependence of the oxygen contents on the time of etching as well as its distribution in layers are shown in Fig. $3 \mathrm{~b}$. The growth of time of etching results also in increase of the oxygen content (curves 1 to 3 ). However, its distribution over the layer depth is smoother than for $\mathrm{SiO}_{\mathrm{x}}$ complexes. At the same time, with time of etching higher than $5 \mathrm{~min}$ the contents of oxygen on a surface and in depth of a layer practically does not change (curve 4). This distribution of oxygen is probably related to a deeper etching of surface defects on silicon substrates. All the studied nanostructured $\mathrm{Si}$ layers contain from 20 up to 50 at.\% of carbon (Fig. 3c). However, its distribution in depth of a layer depending on time of nanostructured $\mathrm{Si}$ formation significantly differs from similar dependences for ions of oxygen and 
$\mathrm{SiO}_{x}$ complexes. As it is seen from Fig. 3c, reduction in the concentration of carbon is observed for increased times of etching (curves 1 to 3 ), and at times of etching exceeding $5 \mathrm{~min}$ its contents and distribution in depth practically do not change (curve 4). The concentration distributions obtained using the AES method correlate well with SIMS results.

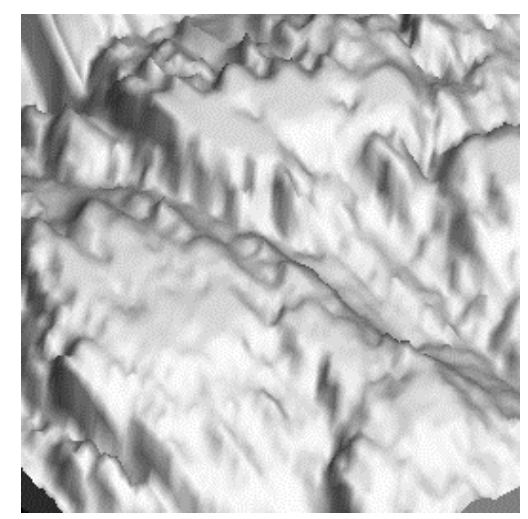

Fig. 2. STM images of the nanostructured Si surface (scanned area is $40 \times 40 \mathrm{~nm}^{2}$ ).
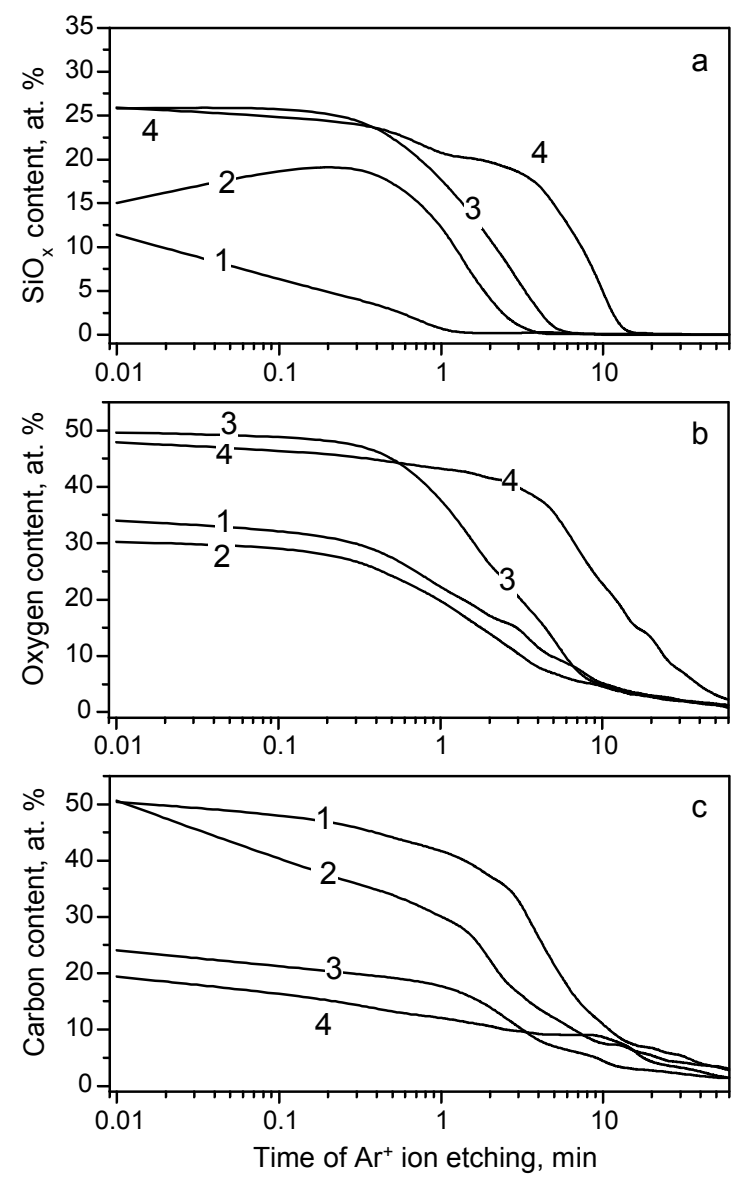

Fig. 3. Depth distribution of elements in nanostructured silicon by using AES, time of stain etching: 1.5 (1), 2 (2), 4 (3), and $5(4) \mathrm{min}$.

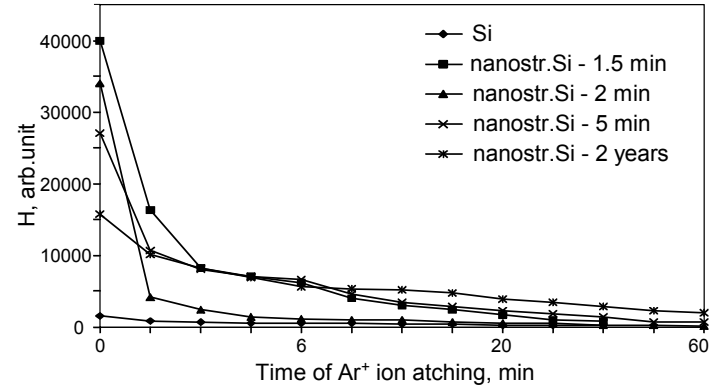

Fig. 4. Depth distribution of hydrogen in nanostructured silicon obtained by means of secondary ion-mass spectroscopy.

Besides, this SIMS method carries additional information on the content of hydrogen, to which the AES method is not sensitive. It is seen from Fig. 4 that at chemical etching the content of hydrogen in the surface area (curves 2 to 4 ) and its stable preservation essentially grows for a long time (curve 5). Stable hydrogenation of the surface layer should provide passivation of the surface recombination centers and allow creating photodetectors with a high sensitivity to ultra-violet and visible radiation.

\subsection{Photoelectric and photoluminescent characteristics}

In the course of nanostructured $\mathrm{Si}$ formation on substrates from monocrystalline silicon, there are changes not only in structural properties, that results in changes of the bandgap width and occurrence of quantum size effects, but also in formation on a surface of new silicon compounds with the increased content of hydrogen and amorphous silicon. This complex structure results in occurrence of new electrophysical, photoelectric, thermophysical, electro- and photoluminescent properties, which allows creating some new types of semiconductor devices, in particular, photodetectors for the short-wave part of a spectrum. Variety of electrical properties of nanostructured $\mathrm{Si}$ and its contacts to a metal electrode and silicon substrate result in an experimentally observable wide set of the characteristics corresponding to sandwichlike structures with nanostructured $\mathrm{Si}$ layers. It is established that maximal photosensitivity in a visible spectral range is characteristic for nanostructured Si layers with the thickness of 10 to $15 \mathrm{~nm}$ (Fig. 5). With growth of thickness, photosensitivity of nanostructured $\mathrm{Si}$ layer decreases. At the same time, the maximal sensitivity to ultra-violet radiation is obtained for nanostructured $\mathrm{Si}$ layers with the thickness 20-25 nm (Fig. 6).

The reduction in the factor of photosensitivity for the samples with increased time of etching is caused by increase in the size of pores and, accordingly, by increase in resistance, however the factor of sensitivity to ultra-violet radiation grows. The latter is explained by occurrence of quantum-size effects resulting in quantization of a power spectrum of carriers of the charge and, as a consequence, in the increase of the bandgap width. 


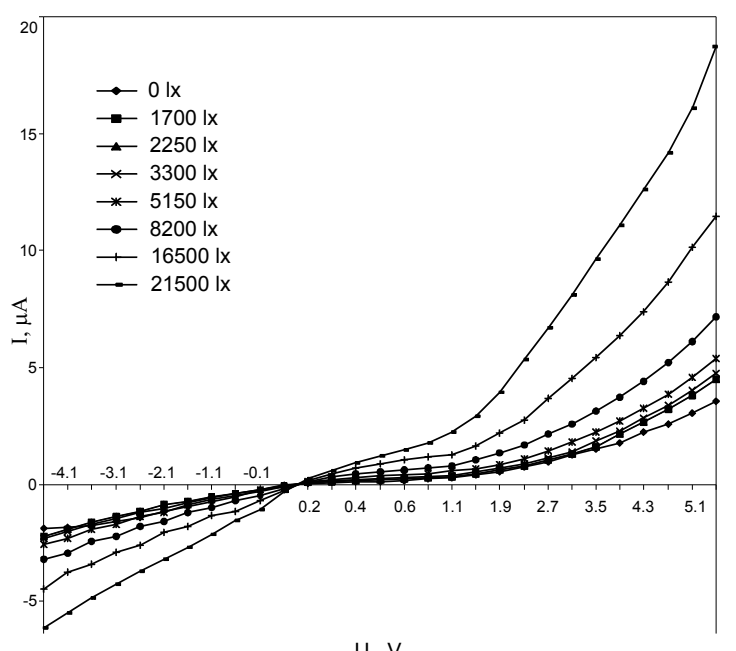

U, V

Fig. 5. Light and dark current-voltage characteristics of the nanostructured Si layers.

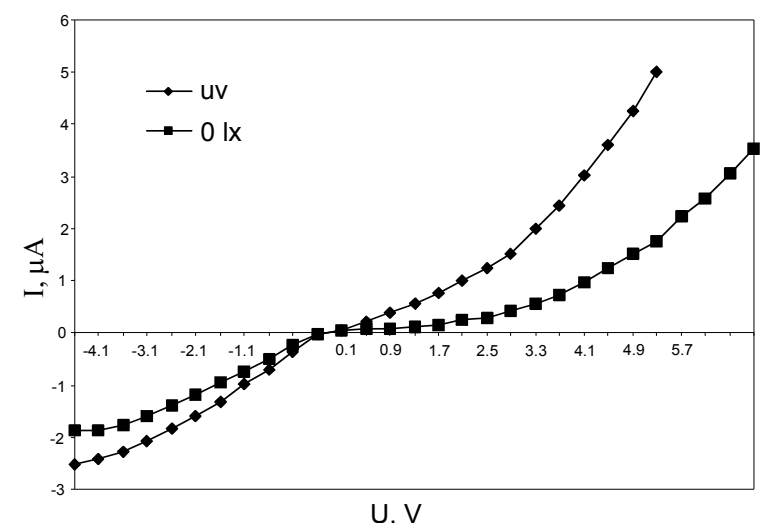

Fig. 6. Current-voltage characteristics for the nanostructured layers under UV illumination.

For diode structures, reduction of the rectification factor with increase of time of etching is observed, which is also caused by increase in the size of pores and, accordingly, by increase of resistance.

Our researches were also focused on study of catalytic and adsorption characteristics of nanostructured $\mathrm{Si}$ for creation of a selective sensor suitable for determination of the gas concentration and humidity, sensor controlling the pressure and force, biosensor for determination of organic pollutions, concentration of complex organic substances, medicinal means, toxic substances on the basis of change of the optical, photoluminescence and photoelectric characteristics. Perspective is application of nanostructured $\mathrm{Si}$ as operation elements for biochemical and biological sensor controls. It became especially urgent after it was shown that nanoporous silicon is a biocompatible material. The photoluminescent spectra of nanostructured Si layers obtained using monocrystalline silicon are characterized by a peak located at the wavelengths about 650 to $670 \mathrm{~nm}$. In the case of formation of nanostructured $\mathrm{Si}$ layers on metallurgical silicon, the obtained spectral dependences of all the samples are characterized by much more intensive photoluminescence. In most cases, photoluminescence intensity has values significantly exceeding those in spectra of nanostructured Si formed on monocrystalline silicon.

Thus, it is necessary to note that, irrespective of modes providing formation of nanostructured Si layers, the position of a spectral maximum is invariable and is precisely fixed at $680 \mathrm{~nm}$. Therefore, in our further experiments samples with nanostructured $\mathrm{Si}$ layers prepared on substrates from metallurgical silicon were used. To study changes in photoluminescence properties of nanostructured $\mathrm{Si}$, pesticides phosalone was chosen. For fixation of toxin, the gel with ferment immobilizing this toxin butyrylcholin esterase was used. Construction of a breadboard model of the photoluminescent sensor of biological substances and molecular pollution on nanostructured $\mathrm{Si}$ includes a source of a ultra-violet radiation with the wavelength of radiation $350 \mathrm{~nm}$, two photodetectors located under an angle $20-25^{\circ}$ in relation to the plane of a plate with nanostructured Si layer, and photodetector for measurements of falling ultra-violet radiation. During the measurement, the surface of the sensor with nanostructured Si layer was closed by a silica glass transparent for ultra-violet radiation. After adsorption of biomolecules, transmission of this glass is worsened, the size of a falling flow of ultra-violet radiation decreases, the value of photoluminescence and output voltage of photodetectors is reduced. The output signal for measurements of a level of pollution represents the ratio of output signal from photodetectors located under an angle to output signal of the photodetector measuring falling ultra-violet radiation. This construction is typical for sensor systems of a differential type and allows determining the molecular pollution of optical systems. Using this sensor, the dependence of changes in the output voltage on the concentration of phosalone was measured (Fig. 7). When determining the concentration of toxin phosalone by using the method of disbalance of the bridge, where a sample with nanostructured Si layer was used as the sensitive element in the measuring channel, it was established the possibility to determine such a small concentration of pesticides as $10^{-8}-10^{-9} \mathrm{~mol} / \mathrm{l}$, which is hardly possible by any other methods, except for proposed in this work.

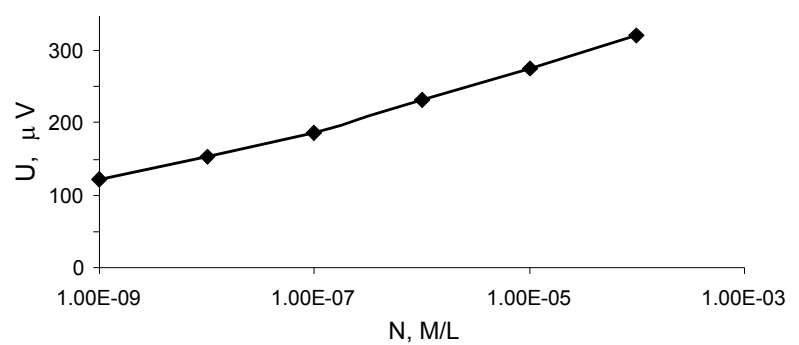

Fig. 7. Dependence of the disbalance signal for photoluminescent biosensor of differential type on the phosalone concentration. 
Thus, biosensor controls on samples with nanostructured Si layers can be successfully used for determination of specific biological pollutions such as protein components, polysaccharides, cells used during biotechnological synthesis.

\section{Conclusions}

In this work, nanostructured layers $(3-60 \mathrm{~nm})$ on substrates of very large $\left(100 \mathrm{~cm}^{2}\right)$ monocrystalline silicon, multicrystalline silicon and metallurgical silicon have been fabricated by the method of stain etching. It has been shown that, during formation of nanocrystalline silicon layer on a surface of monocrystalline silicon, both content and distribution of oxygen and oxidized silicon change. Besides, content of hydrogen at the surface of layers essentially grows. The dependence of the hydrogen content on time of etching and stability of its preservation during a long period of time have been ascertained. This stable hydrogenation of surface layers of silicon provides passivation of the surface recombination centers.

Researches of properties of nanostructured $\mathrm{Si}$ prepared using the method of chemical processing have confirmed an opportunity to create this multifunctional material with stable characteristics, to preset these characteristics, to create high-sensitive photodetectors of visible and ultra-violet radiation, which surpass by their parameters all known analogs.

It has been shown that the biosensors with nanostructured $\mathrm{Si}$ layers can be successfully used to determine small concentrations of toxins (pesticides phosalone $10^{-8}-10^{-9} \mathrm{~mol} / \mathrm{l}$ ) as well as for specific biological pollutants, such as protein components, polysaccharides, cells used during biotechnological synthesis.

\section{References}

1. S.V. Svechnikov, A.V. Savchenko, G.A. Sukach, A.M. Evstigneev, E.B. Kaganovich, Light-emitting layers of porous silicon: preparation, properties and application // Optoelektronika I poluprovodnikovaya technika, 27, p. 3-29 (1994), in Russian.

2. Hiroshi Tayanaka, Kazushi Yamauchi, Takeshi Matsushita, Thin-film crystalline silicon solar cells obtained by separation of a porous silicon sacrificial layer // Proc. $2^{\text {nd }}$ World Conf. on Photovoltaic Solar Energy Conversion, Vienna, July 6-10, 1998, p. 1273-1277.

3. E.A. Tutov, E.N. Bormontov, V.M. Kashkarov, M.N. Pavlenko, E.P. Domashevskaya, Influence of water vapor adsorption on the $\mathrm{C}-\mathrm{V}$ characteristics of heterostructures containing porous silicon // Technical Physics, 48(11), pp. 1442-1448 (2003).

4. N.F. Starodub, V.M. Starodub, Biosensors based on the photoluminescence of porous silicon. Overall characteristics and application for medical diagnostics // Sensor Electronics and Microsystem Technologies, 2, p. 64-79 (2004).

5. Y.M. Weng, Z.N. Fan, X.F. Zong, Luminescence studies on porous silicon // Appl. Phys. Lett., 63(2), p. $168-170$ (1993).

6. A.I. Luchenko, M.M. Melnichenko, K.V. Svezhentsova, Chemical modification of single-crystal silicon surface // Journal of Optoelectronics and Advanced Materials, 9(5), p. 1431-1434 (2007). 